HEALTH PSYCHOLOGY REPORT $\cdot 2020$

ORIGINAL ARTICLE
Luot V. Nguyen (D)

$1 \cdot \mathrm{A}, \mathrm{B}, \mathrm{C}, \mathrm{D}, \mathrm{E}, \mathrm{G}$

Thu H. Tran

$1 \cdot \mathrm{A}, \mathrm{E}, \mathrm{F}$

Thu T. A. Nguyen

$1 \cdot A, D, E$
Dat B. Nguyen

$1 \cdot \mathrm{A}, \mathrm{D}, \mathrm{E}$

Harriot Beazley

$2 \cdot \mathrm{A}, \mathrm{E}, \mathrm{F}$

Mai T. T. Giang

$3 \cdot \mathrm{A}, \mathrm{E}, \mathrm{F}$

\title{
Exposure to sexually explicit Internet material among adolescents: a study in Vietnam
}

\section{BACKGROUND}

With the influence of Confucian culture, sex is often considered a taboo subject in Vietnam. This study aimed to examine the prevalence of exposure to sexually explicit Internet material (SEIM) among adolescents in Vietnam, as well as the factors affecting exposure to SEIM.

\section{PARTICIPANTS AND PROCEDURE}

The sample consisted of 886 Vietnamese adolescents ( $58.10 \%$ female) aged 15 to 18 years $(M=16.72, S D=0.72)$. The survey was adapted from the study of Van Ouytsel, Ponnet and Walrave to assess the frequency of exposure to SEIM. Several measurement scales of factors affecting exposure to SEIM among adolescents were used including the Perceived Realism of SEIM, the Sexual Sensation Seeking Scale, the Family Cohesion and the Parental Monitoring Scale.

\section{RESULTS}

The overall prevalence of exposure to SEIM was $84.10 \%$, with a gender difference: $89.80 \%$ in males and $80.00 \%$ in females. The prevalence of passive exposure to SEIM was $58.30 \%$, higher than $41.70 \%$ for active exposure. Perceived realism of SEIM, sexual sensation seeking and Internet usage time for entertainment are factors affecting frequencies of exposure to SEIM among adolescents, with $\beta$ coefficient values of $.29, .18$ and .16 respectively.

\section{CONCLUSIONS}

The majority of children in this study reported SEIM exposure. Parents, schools and other stakeholders should pay attention to sex education for children early on, diversifying forms and subjects of sex education. The content of sex education should emphasize the responsibility in the decisions made by children related to sex.

\section{KEY WORDS}

adolescent; internet; pornography; sexually explicit internet material; exposure to SEIM

ORGANIZATION - 1: University of Social Sciences and Humanities, Vietnam National University, Hanoi, Vietnam .

2: University of the Sunshine Coast, Sippy Downs, Australia · 3: Vietnam Academy of Social Sciences, Hanoi, Vietnam AUthors' CONTRIBUtions - A: Study design - B: Data collection - C: Statistical analysis - D: Data interpretation .

E: Manuscript preparation · F: Literature search · G: Funds collection

CORRESPonding AUthor - Luot V. Nguyen, Ph.D., University of Social Sciences and Humanities, Vietnam National

University, 336 Nguyen Trai, Thanh Xuan, 084 Hanoi, Vietnam, e-mail: luotnv@vnu.edu.vn

to Cite this ARTICle - Nguyen, L. V., Tran, T. H., Nguyen, T. T. A., Nguyen, D. B., Beazley, H., \& Giang, M. T. T. (2020).

Exposure to sexually explicit Internet material among adolescents: a study in Vietnam. Health Psychology Report.

https://doi.org/10.5114/hpr.2020.99394

RECEIVED 03.06.2020 • REVIEWED 20.08.2020 • ACCEPTED 27.08.2020 • PUBLISHED 28.09.2020 


\section{BACKGROUND}

In Vietnam, sexual education is a topic that is not discussed at home, and is avoided and not treated in a balanced way at school (Khuat, Bach, \& Nguyen, 2009). For this reason, peers become the ultimate advisers and role models for young people wishing to understand sex (Khuat et al., 2009). Most studies of sexuality in Vietnam are approached from the perspective of public health or health care issues, and have focused on studying adult sexuality. Adolescent sexuality has not received proper attention due to the widespread notion that sex is taboo among young people. However, due to the rapid growth of the Internet, adolescents have been increasingly exposed to sexually explicit Internet material (SEIM) in recent years. As a result of many barriers, including cultural norms, the delicate subject matter, shyness, and a lack of sexual knowledge, parents often avoid providing sexual education to their children. Consequently, the Internet has become an alternative source of information on sexual issues, which is not usually available from other sources, such as family and school (Ngo, Ross, \& Ratliff, 2009; Trinh, 2004).

Vietnam is one of the countries with the largest number of Internet users, and also the country with the fastest growth in the number of people using social media in the world (Internet World Stats, 2019). In Vietnam, Wi-Fi has been popularized in different spaces such as entertainment centers, restaurants, and Internet games centers. A high percentage of young people in Vietnam use or own a smartphone. According to TNS, in 2013, 58\% of Vietnamese people aged 16 to 24 years used a smartphone (TNS, 2014). According to Cooper (1998), the Internet has had an impact on human sexual behavior due to the Internet's three benefits of anonymity, accessibility and affordability (Cooper, 1998). Added to this, adolescents' natural curiosity to explore new things, together with good telecommunications infrastructure, has increased the possibility of exposure to SEIM among adolescents in Vietnam (Truong, 2013).

In some ways, SEIM has a positive impact as people of most ages can gain sexual knowledge and skills through watching these materials (Alexy, Burgess, \& Prentky, 2009). The negative effects on minors are, however, of concern as adolescents who use the Internet without adult supervision cannot distinguish and be aware of unsafe and unhealthy Internet content (Owens, Behun, Manning, \& Reid, 2012; Peter \& Valkenburg, 2016). The psychological impact of SEIM on adolescents is extensive, including sexual attitudes, beliefs and behaviors, sometimes resulting in risky sexual behavior (Owens et al., 2012; Peter \& Valkenburg, 2016). There is ample evidence that exposure to SEIM is closely related to permissive sexual attitudes and traditional gender roles in sex (Lo \& Wei, 2005; Peter \& Valkenburg, 2006a); risky sexual behaviors such as not using condoms during sex, having multiple sex partners at the same time, and sexual intercourse at an early age (Peter \& Valkenburg, 2011a; Weber, Quiring, \& Daschmann, 2012).

Theoretically, studies on the topic of exposure to SEIM among adolescents are well known in Western cultures, with the majority of research being conducted in Europe, the U.S.A. and Australia (Peter \& Valkenburg, 2016). Meanwhile, the understanding of adolescent exposure to SEIM in Africa, the Middle East, Russia and Southeast Asia (including Vietnam) is very limited. To date there has been no study published in peer-reviewed journals on this topic in Vietnam. A study on the prevalence and impact of Vietnamese adolescents' exposure to SEIM is, therefore, important.

Regarding SEIM, notable are the concepts of BraunCourville and Rojas (2009) and Peter and Valkenburg (2011a). Braun-Courville and Rojas emphasize sexually explicit websites and define them as "X-rated or pornographic Web sites that either: describe people having sex, show clear pictures of nudity or people having sex, or show a movie or audio that describes people having sex" (p. 157).

Subsequently, the study of Peter and Valkenburg (2011a) provided a relatively detailed definition of SEIM as "professionally produced or user generated pictures or videos (clips) on or from the Internet that are intended to arouse the viewer. These videos and pictures depict sexual activities, such as masturbation as well as oral, anal, and vaginal penetration, in an unconcealed way, often with a close-up on genitals" (p. 1015).

In this study, taking into account the views of previous authors, especially those of Peter and Valkenburg (2011a), we define SEIM as websites containing texts, images, audio, video clips or films that create or elicit and/or enhance sexual feelings, thoughts or behaviors in the recipient. Exposure to SEIM is defined as the behavior of being exposed to websites containing texts, images, audio, video clips or films that create or elicit and/or enhance sexual feelings, thoughts or behaviors in the recipient. The exposure can take place either actively or passively, through electronic devices with an Internet connection (e.g. smartphone, tablet, computer). Exposure to SEIM in this study did not include offline materials such as sexually suggestive magazines, sexual and reproductive health guidelines, CDs and videos containing pornography. Given the prevalence of Wi-Fi coverage in Vietnam, the exposure to SEIM in this study does not clearly define whether the exposure takes place in families, schools and public spaces since the actual exposure of teenagers may occur in any of the above-mentioned spaces.

Adolescents are in the age of transition from childhood to adulthood that involves many crisis-causing developments and is often known as the rebellious 
phase with many dramatic physical and psychological changes (Truong, 2013). However, due to their developmental changes, they also often face difficulties and crises in learning, relationships with parents and peers. One significant psychological characteristic is that the children's self-awareness is heightened since adolescents tend to focus on defining their own personal values and goals and establishing their autonomy from the family (Berk, 2017). Adolescents have a thirst for affirming themselves, but sometimes they cannot distinguish right from wrong due to limited life experience. Therefore, it is easy for them to become involved in negative behaviors if there is no guidance from parents, teachers or their own peers.

In Vietnam, there is no clear delineation of children and adolescents. Article 1 of Vietnam's Law on Children states that "children are under 16 years old" (SRV, 2016). According to the World Health Organization, adolescence is the transition period between childhood and adulthood, and 'adolescents' are individuals in the 10-19 years age group (WHO, 2018). In this study, the term 'adolescent' is used to define individuals in the 15-18 years age group who are studying in grades 10-12 at public high schools.

In the literature, exposure to SEIM has often been studied with a focus on either the prevalence of exposure to SEIM, and the predictors or affecting variables of exposure to SEIM among adolescents. In the group of adolescents who were exposed to SEIM passively (i.e. they were passively or inadvertently exposed to SEIM from various sources on the Internet when using electronic devices), the prevalence rates range widely. In a study of adolescents in the U.S.A., Wolak, Mitchell, and Finkelhor (2007) found that $19.00 \%$ of teenagers aged 10-12 years are exposed to SEIM passively. In Australia, the prevalence is $60.00 \%$ in boys and $84.00 \%$ in girls at the age of 16 to 17 (Flood, 2007), and even up to $87.00 \%$ in recent research of Australian adolescents (Lim, Agius, Carrotte, Vella, \& Hellard, 2017). Similar findings were found in research of adolescents in Greece, with $40.00 \%$ and $27.00 \%$ among boys and girls aged 10-14 years, and $44.00 \%$ and $28.00 \%$ among boys and girls aged 15-18 years, respectively (Tsaliki, 2011). Findings from Chen, Leung, Chen, and Yang (2013) revealed that $41.30 \%$ of Taiwanese teenagers in grades 10 to 12 were exposed to SEIM. In Switzerland, $47.00 \%$ of boys and $35.00 \%$ of girls less than 18 years of age were inactively exposed to SEIM (Luder et al., 2011).

In the group of teenagers who were exposed to SEIM actively (i.e. they actively seek to access websites on the Internet to engage with SEIM), the prevalence rates vary widely. For instance, Ybarra and Michell (2005) found the rate of only about $8.00 \%$ of adolescents aged 10-17 in the U.S., while the prevalence among Taiwanese adolescents in grades 10 to 12 was $59.00 \%$ (Chen et al., 2013). Studies of teenagers in European countries revealed the SEIM expo- sure prevalence of $18.00 \%$ in Sweden, $20.00 \%$ in the Netherlands and $46.00 \%$ in Belgium (Skoog, Stattin, \& Kerr, 2009; Vandenbosch \& Eggermont, 2013).

In the previous literature, predictors of exposure to SEIM among adolescents can be generalized into individual-related variables such as gender, age, sexual orientation, personality characteristics, social norms, biological development, sexual experiences; and variables related to adolescents' relationship with their family and peers.

Several studies have shown that the active exposure to SEIM among adolescents is related to gender (Lopez, Mukaire, \& Mataya, 2015; Luder et al., 2011; Peter \& Valkenburg, 2006a). Boys in these studies were more exposed to SEIM than girls. This relation is evident in both Western and Eastern cultures. For instance, $71.00 \%$ of males and $40.00 \%$ of females in the Netherlands were actively exposed to SEIM (Peter \& Valkenburg, 2006a). The rates for boys and girls were $38.00 \%$ and $2.00 \%$ respectively in Australia (Flood, 2007), $40.00 \%$ and $13.00 \%$ respectively in the U.S.A. (Bleakley, Hennessy, \& Fishbein, 2011), and $29.00 \%$ and $1.00 \%$ respectively in Switzerland (Luder et al., 2011). Similar differences were also prevalent among teenagers in Asian countries. A study of Cambodian adolescents showed that $20.40 \%$ of boys and $1.50 \%$ of girls were actively exposed to SEIM (Lopez et al., 2015).

Sexual orientation is another predictor of exposure to SEIM. Adolescents with a bisexual sexual orientation and male homosexuals were more exposed to SEIM than heterosexual counterparts (Luder et al., 2011; Peter \& Valkenburg, 2011a). Adolescents with a homosexual orientation tend to watch SEIM during a sex party or aberration party. They watch anal sex scenes with and without using condoms. Adolescents with a heterosexual or bisexual orientation tend to watch vaginal sex scenes with or without using condoms (Downing, Schrimshaw, Scheinmann, AntebiGruszka, \& Hirshfield, 2017). Sexual suggestive materials including SEIM play an important role in the lives of black youths with a homosexual orientation, and are the only source of information for them to learn about sexual development (Arrington-Sanders et al., 2015).

Regarding personality characteristics, the literature reveals that adolescents with a strong sense of adventure, sensation seeking, low self-esteem and less satisfaction with life were more exposed to SEIM than other teenagers (Beyens, Vandenbosch, \& Eggermont, 2014; Holt, Bossler, \& May, 2012; Kim, 2001; Kim, 2011; Luder et al., 2011; Peter \& Valkenburg, 2011a; Ševčíková, Šerek, Barbovschi, \& Daneback, 2014). According to Efrati and Amichai-Hamburger (2020), higher degrees of exposure to SEIM are also found among adolescents with higher scores in Extraversion and Neuroticism and lower scores in Conscientiousness and Agreeableness. They also pointed
Exposure to SEIM among adolescents 
Luot V. Nguyen,

Thu H.Tran,

Thu T. A. Nguyen,

Dat B. Nguyen,

Harriot Beazley,

Mai T. T. Giang out that teenagers with narcissism also engage with SEIM more than those with less of this personality expression (Efrati \& Amichai-Hamburger, 2020). Youths who have higher scores of impulsiveness or often respond to social desirability rather than to their true self are more involved in exposure to SEIM (Efrati \& Amichai-Hamburger, 2020). Furthermore, those who are more aware of the perceived realism of SEIM are more engaged in SEIM (Peter \& Valkenburg, 2006b).

Some studies have found a positive relationship between the Internet usage time for entertainment and the exposure to SEIM among adolescents. Specifically, a higher volume of time spent on the Internet indicated a higher degree of exposure to SEIM in the teenage population (Mitchell, Finkelhor, \& Wolak, 2003; Rasmussen, Ortiz, \& White, 2015).

Regarding variables related to social norms, studies have revealed that teenagers who tend to disrupt rules and social norms, and those who use substances are more likely to be exposed to SEIM than their counterparts (Wolak et al., 2007; Ybarra \& Mitchell, 2005). Other studies (Hardy, Steelman, Coyne, \& Ridge, 2013; Mesch, 2009; Rasmussen \& Kohut, 2019) revealed that adolescents with religious involvement (e.g. Catholic, conservative Protestant, mainline Protestant) tend to be less exposed to SEIM than those without any religion.

In regards to age, puberty and sexual experience, some investigations have found a close association between these variables with the degree of exposure to SEIM among adolescents, though findings on the link of age and puberty to SEIM exposure remain inconclusive. Some studies revealed a positive relation between the exposure to SEIM and age (Ševčíková et al., 2014; Shek \& Ma, 2012; Wolak et al., 2007; Ybarra \& Mitchell, 2005) or with sexual experience, especially among girls (Ševčíková et al., 2014). Other examinations found no differences in SEIM exposure associated with ages (Holt et al., 2012; Mesch, 2009; Peter \& Valkenburg, 2006b, 2011a, b) or with sexual experience (Peter \& Valkenburg, 2006a). In contrast, findings that biological maturity is closely related to SEIM exposure are consistent in the empirical literature. The sooner the teenagers start their puberty, the greater exposure to SEIM they have (Beyens et al., 2014; Luder et al., 2011).

The relationship with family was found to be closely related to teenage exposure to SEIM. Adolescents with little family cohesion (Mesch, 2009), less functioning family responsibilities (Shek \& Ma, 2014) and lack of support from families had higher levels of SEIM exposure than the other teenage groups (Shek \& Ma, 2012). Similar results are found with those who are in families with little emotional attachment (Ybarra \& Mitchell, 2005), or with parents or caregivers using imposed education; or those experiencing family conflicts and less communication between members (Ma \& Shek, 2013). In contrast, the frequency of SEIM exposure decreased among adolescents in families with parents being open, talking with children about sexuality in general, and SEIM in particular (Peter \& Valkenburg, 2006a; Wolak et al., 2007), or those with parental monitoring including parents' understanding of where their children often go, the activities and friends their children have (Tomic, Buric, \& Stulhofer, 2018), or with parents setting rules and standards of conduct for children, and having plans to manage the safe use of the Internet (Rasmussen et al., 2015).

In terms of relationships with peers, exposure to SEIM increases among adolescents whose majority of friends are younger than them and with whom they frequently share and talk about sexual suggestive materials (Peter \& Valkenburg, 2016). Further, adolescents who have experienced physical or sexual abuse or have negative life experiences such as divorced parents, traditional bullying or online bullying are more likely to be exposed to SEIM (Shek \& Ma, 2014). Similar observations are made among teenagers with a lack of attachment to school and family, or those with less social intimacy (Efrati \& Amichai-Hamburger, 2020).

In Vietnam, most sexual research is approached primarily from the perspective of public health or health care. In particular, previous studies in Vietnam have often revolved around reproductive health and abortion issues for women (Bélanger \& Hong, 1999). In recent years, some studies have focused on sexual satisfaction in marriage relationships (Do \& Barh, 2017; Do, Khuat, \& Nguyen, 2017); gender diversity and sexuality (Nguyen, 2018); culture of sexuality (Pham, 2018); transgender and cultural and social barriers (Nguyen, 2019). Studies with young people have revealed sexual practices among young people (Duong et al., 2004; Ngo et al., 2009), communication among and sexual satisfaction of young people (Nguyen, Bui, \& Le, 2017), and the sexual lives of young Vietnamese (Bui \& Nguyen, 2018). Notably, one study among young people aged 15-19 years suggested that adolescents in Hanoi use the Internet as an alternative source of information on sexual issues, which is not available from other sources such as family and school (Duong et al., 2004; Ngo et al., 2009). The study also found that young people used the Internet as a means to express their sexual perception and desire.

In a study of attitudes toward sexting, subjective norms towards sexting, behavioral intentions and sexting behaviors among secondary school pupils and undergraduate students, Tran and Nguyen (2016) found significant differences in sexting behaviors between males and females. The estimated prevalence of participants' sexting was $15.00 \%$. Also, attitudes toward sexting, subjective norms toward sexting, and sexting behavioral intentions were found to pre- 
dict lifetime and current sexting behaviors, with behavioral intentions as the strongest predictor sexting behaviors (Tran \& Nguyen, 2016).

The literature review reveals that exposure to SEIM among adolescents has attracted the attention of numerous researchers around the world. The major research focus has been on the prevalence of SEIM exposure among adolescents, and the predictors of adolescent exposure to SEIM in relation to individual-related variables and the adolescent relationship with family and friends. The variables predicting the SEIM exposure among adolescents, however, remain inconclusive and need further investigation. Further, the studies were largely conducted on adolescent samples in Western countries. There is a dearth of studies on this topic in Southeast Asian cultures, including Vietnam. This study therefore aimed to describe the prevalence and the predictors of exposure to SEIM among Vietnamese adolescents. In this study, the following three hypotheses are formulated:

H1. Exposure to SEIM among adolescents is common.

H2. Younger children, children in early puberty, sexual sensation seeking, Internet usage time for entertainment, and perceived realism of SEIM positively predict exposure to SEIM among adolescents.

H3. Life satisfaction, family cohesion, and parental monitoring negatively predict exposure to SEIM among adolescents.

\section{PARTICIPANTS AND PROCEDURE}

\section{PARTICIPANTS}

A convenient sampling strategy was employed to select respondents in the provinces of Hanoi, Hai Phong, Binh Dinh and Binh Phuoc in Vietnam. Data collection was conducted from August to October 2019. Adolescent participants in this study attended public high schools in their localities.

The total number of participants was 886 adolescents $(58.10 \%$ female), aged from 15 to 18 , with the average age of $16.72(S D=0.72)$. Regarding sexual orientation, 756 participants $(85.30 \%)$ reported a heterosexual orientation, $96(10.80 \%)$ a bisexual orientation, $16(1.80 \%)$ a homosexual orientation, and $18(2.00 \%)$ an asexual orientation. For the current romantic love relationship, 289 (32.60\%) participants reported having been in love, $170(19.20 \%)$ reported being in a love romantic relationship, $409(46.20 \%)$ reported never having loved anyone, and $18(2.00 \%)$ did not respond. $752(84.90 \%)$ respondents were living with their parents and $754(85.30 \%)$ owned a smartphone. The average Internet usage time for entertainment was reported as 4.39 hours per day. 123 (13.90\%) participants reported religious involvement, and 763 (86.10\%) reported no religious affiliation.

\section{MEASURES}

On the basis of the literature review, we determined that the dependent variable is exposure to SEIM, and the eight independent variables are: age (Ševčíková et al., 2014; Shek \& Ma, 2012; Wolak et al., 2007); puberty age (Beyens et al., 2014; Luder et al., 2011; Peter \& Valkenburg, 2011b); Internet usage time for entertainment (Mitchell et al., 2003; Rasmussen et al., 2015); life satisfaction (Peter \& Valkenburg, 2006b, 2011a); perceived realism of SEIM (Peter \& Valkenburg, 2006b); family cohesion (Mesch, 2009; Peter \& Valkenburg, 2016); sexual sensation seeking (Peter \& Valkenburg, 2006b); and parental monitoring (Tomic et al., 2018; Ybarra \& Mitchell, 2005).

\section{Dependent variable: exposure to SEIM}

A question was adapted from Van Ouytsel, Ponnet and Walrave (2014) to examine exposure to SEIM among adolescents. Van Ouytsel et al. (2014) investigated the frequencies of exposure to pornography among Belgium adolescents by asking the question "How often do you watch pictures or videos in which naked men or women are depicted (e.g. magazines such as Playboy or a pornographic Web site?", with response options range from 1 (never), 2 (a few times a year or less), 3 (once a month), 4 (a few times a month), 5 (1-3 times a week), 6 (3-6 times a week), 7 (every day) to 8 (more than 2 times a day) (Van Ouytsel et al., 2014).

The question was adjusted in our study to a 6-response scaled question only focusing on exposure to SEIM in a 6-month period. Our question was "Please specify your frequencies of exposure to sexually explicit Internet materials in the last 6 months?". Response alternatives range from 1 (none), 2 (less than once a month), 3 (1 to 3 times a month), 4 (weekly), 5 (several times a week) to 6 (daily), with a higher score indicating a higher degree of exposure to SEIM.

\section{Independent variables}

1. Age of children. Children reported their ages, which range from 15 to 18 .

2. Internet usage time for entertainment (in hours). Calculated by the number of hours that children used the Internet for entertainment.

3. Puberty age. Children reported their puberty age.

4. Life satisfaction. In measuring Vietnamese adolescents' satisfaction with their life, we designed a question, specifically "How satisfied are you with your own life?". The question was assessed in the form of a 5-point Likert type scale ranging from 1 (completely dissatisfied), 2 (dissatisfied), 3 (less satisfied), 4 (satisfied) to 5 (very satisfied). This question was used in the study by Mai, Luot, Beazley, and Dat (2019) on a sample group of Vietnamese adolescents.
Exposure to SEIM among adolescents 
Luot V. Nguyen,

Thu H. Tran,

Thu T. A. Nguyen,

Dat B. Nguyen,

Harriot Beazley,

Mai T. T. Giang
5. Perceived realism of SEIM. The Perceived Realism of SEIM Scale (Peter \& Valkenburg, 2006b) was also used. The scale consisted of 4 items, for example, "I find sex on the Internet realistic", with response options ranging from 1 (strongly disagree), 2 (disagree), 3 (confused) 4 (partially agree) to 5 (totally agree). The perceived realism of SEIM was calculated by computing 4 variables and dividing by the total number of variables, with a higher score presenting a higher level of perceived realism of SEIM (Peter \& Valkenburg, 2006b). In this study, Cronbach's $\alpha$ was .79.

6. Family cohesion. The Family Cohesion scale (de Graaf, van de Schoot, Woertman, Hawk, \& Meeus, 2012) consists of 3 items, for example "In our family, everybody minds their own business", and answer options range from 1 (totally not true) to 4 (totally true). Items are redeemed before computing, with a higher score describing a higher level of family cohesion (de Graaf et al., 2012). In this study, Cronbach's $\alpha$ was .61.

7. Sexual sensation seeking. The Sexual Sensation Seeking Scale (Kalichman \& Rompa, 1995) was also adapted for this study. The scale consists of 10 items, for example, "I am interested in trying out new sexual experiences", with 4 answer alternatives for each item, including 1 (not like me at all), 2 (like me a little), 3 (pretty like me), 4 (completely like me). The scale score was calculated by computing 10 items and divided by the total number of items, with a higher score indicating a higher level of sexual sensation seeking (Kalichman \& Rompa, 1995). In this study, Cronbach's $\alpha$ was .92.

8. Parental monitoring. The Parental Monitoring Scale (Wight, Williamson, \& Henderson, 2006) consists of 4 items, for example "Do you have to ask permission to go out in the evening?", with 4 response options ranging from 1 (never), 2 (occasionally), 3 (often) to 4 (always). Parental monitoring is calculated by means of four items, with a higher score demonstrating a higher level of parental monitoring (Wight et al., 2006). In this study, Cronbach's $\alpha$ was .75 .

In addition, children's demographic variables such as gender, religion, sexual orientation, romantic relationship status, residence places and phone/laptop ownership were also included in this study.

To ensure the content accuracy, questions for exposure to SEIM among adolescents and measurement scales of perceived realism of SEIM, family cohesion, sexual sensation seeking, and parental monitoring were translated into Vietnamese by an Associate Professor of Psychology and the Vietnamese content was translated into English by a Doctor of Psychology who graduated in Australia and who did not participate in the research group. The question and the scales were then discussed in the research team. Editing of vocabulary and expressions were made to suit children 15-18 years old. In the next step, the questions were conducted with 5 children aged 1518 to test the suitability of the scales and contents. A group discussion between researchers, investigators and children was then organized. The questionnaire was then finalized and a large group survey was conducted.

The Exposure to SEIM scale and other scales show a normal distribution based on skewness and kurtosis indicators, specifically as follows: exposure to SEIM (.78 and -.40$)$, age (.06 and -.32$)$, puberty age $(-.24$ and .42), Internet usage time for entertainment (1.00 and 1.91), life satisfaction (-.40 and .50), perceived realism of SEIM (-.17 and -.81$)$, family cohesion (-.37 and .33), sexual sensation seeking (.97 and -.91$)$, and parental monitoring (-.72 and -.13$)$.

\section{PROCEDURE}

This is a self-report questionnaire survey. At each high school, the research team contacted the School Administration Board and homeroom teachers of the schools. With the consent of the School Administration Board, and homeroom teachers, we introduced the research to the students and called for their voluntary participation. The team also stated the right to anonymity and the right to leave the study if the students feel uncomfortable. Each student was given one questionnaire in a self-completed form. Participants were arranged to sit at a suitable distance to ensure privacy in answering the questionnaire. It took students 15 -20 minutes to complete the questionnaire. The definition of SEIM was recorded at the top of the questionnaire for students to understand before they answered the questions. Participants were not paid for answering the questionnaire and participation was completely voluntary. Representatives of the research team were available to answer any questions or concerns from students.

\section{DATA ANALYSIS}

All the data were processed by SPSS version 23.0. In this study, we conducted descriptive statistics, correlation and multiple regression analyses by the stepwise method.

\section{RESULTS}

\section{PREVALENCE OF EXPOSURE TO SEIM}

The findings are presented in Table 1.

Table 1 indicates that more than 4 out of 5 adolescents in this study, with more males than females, reported having ever been exposed to SEIM. The prev- 
Table 1

Situation of exposure to SEIM among participants

\begin{tabular}{|c|c|c|c|c|}
\hline \multirow[t]{2}{*}{ Classification } & Male & Female & Total & \multirow{2}{*}{$\chi^{2}, t, p$} \\
\hline & \multicolumn{3}{|c|}{$n(\%)$} & \\
\hline \multicolumn{5}{|l|}{ Exposure to SEIM } \\
\hline Yes & $333(89.80)$ & $412(80.00)$ & $745(84.10)$ & \multirow{2}{*}{$\begin{array}{c}\chi^{2}(1,886)=15.34 \\
p<.001\end{array}$} \\
\hline No & $38(10.20)$ & $103(20.00)$ & $141(15.90)$ & \\
\hline \multicolumn{5}{|c|}{ Active or passive exposure to SEIM } \\
\hline Active & $193(58.00)$ & $118(28.60)$ & $311(41.70)$ & \multirow{2}{*}{$\begin{array}{c}\chi^{2}(1,745)=65.08 \\
p<.001\end{array}$} \\
\hline Passive & $140(42.00)$ & $294(71.40)$ & $434(58.30)$ & \\
\hline $\begin{array}{l}\text { Age of first exposure to SEIM } \\
M(S D)\end{array}$ & $13.75(2.15)$ & $14.41(1.75)$ & $14.11(1.96)$ & $\begin{array}{c}t(735)=4.43 \\
p<.001\end{array}$ \\
\hline \multicolumn{5}{|l|}{ Time of exposure to SEIM } \\
\hline 5 minutes or less & $133(41.40)$ & $321(73.60)$ & $445(59.70)$ & \multirow{5}{*}{$\begin{array}{c}\chi^{2}(4,745)=79.17 \\
p<.001\end{array}$} \\
\hline 6-10 minutes & $72(22.40)$ & $44(10.40)$ & $116(15.60)$ & \\
\hline $11-20$ minutes & $46(14.30)$ & $27(6.40)$ & $73(9.80)$ & \\
\hline 21-30 minutes & $48(15.00)$ & $25(5.90)$ & $73(9.80)$ & \\
\hline$>30$ minutes & $22(6.90)$ & $16(3.80)$ & $38(5.10)$ & \\
\hline \multicolumn{5}{|l|}{ Exposure to SEIM with whom? } \\
\hline Alone & $318(85.90)$ & $383(75.10)$ & $701(79.70)$ & \multirow{2}{*}{$\begin{array}{c}\chi^{2}(1,880)=15.57 \\
p<.001\end{array}$} \\
\hline Friends & $52(14.10)$ & $127(24.90)$ & $179(20.30)$ & \\
\hline \multicolumn{5}{|c|}{ Exposure to SEIM types of media } \\
\hline Video clips & $153(51.20)$ & $146(48.80)$ & $299(34.30)$ & - \\
\hline Film & $130(64.40)$ & $72(35.60)$ & $202(23.20)$ & - \\
\hline Photo & $118(43.40)$ & $154(56.60)$ & $272(31.20)$ & - \\
\hline Audio & $19(61.30)$ & $12(38.70)$ & $31(3.60)$ & - \\
\hline Text & $46(21.60)$ & $167(78.40)$ & $213(24.90)$ & - \\
\hline
\end{tabular}

Note. Differences were determined by chi-square test $\left(\chi^{2}\right)$. Reported values are $n(\%)$.

alence of passive exposure was higher than that of active exposure. The average age of first exposure to SEIM among adolescents in this study was 14.11 years, and boys also had earlier exposure than girls. The majority of teenagers reported being exposed to SEIM alone and for 5 minutes or less. The common forms of SEIM to which adolescents were exposed included video clips, photos, texts, films and audio.

\section{PREDICTORS OF EXPOSURE TO SEIM}

Table 2 shows the correlation between exposure to SEIM and the independent variables.

Table 2 shows the correlation between the dependent variable and the independent variables, which varies from -.16 to .38 , and there are 5 pairs of variables with statistically significant correlation coefficients from $p=.05$ to $p=.001$. Specifically, there is a positive correlation between Internet usage time for entertainment, perceived realism of SEIM, sexual sensation seeking and exposure to SEIM. There is a negative correlation between family cohesion and exposure to SEIM among adolescents.

The research team used regression analysis with the stepwise method. Among the 8 independent variables mentioned above, the regression model proposed 3 models: model 1 ( 1 independent variable), model 2 (2 independent variables), and model 3 (3 independent variables).

Testing of the model's appropriateness showed that the statistic $F$ in the three models has a signifi-
Exposure to SEIM among adolescents 
Luot V. Nguyen,

Thu H.Tran,

Thu T. A. Nguyen,

Dat B. Nguyen,

Harriot Beazley,

Mai T.T. Giang

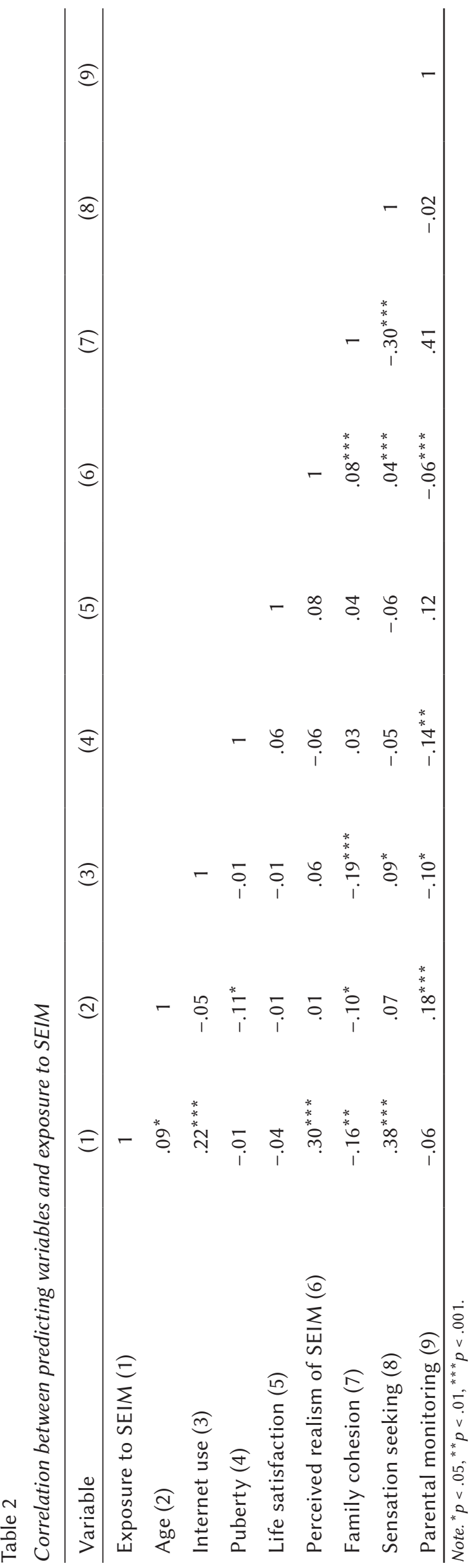

cance level of $p$ from < .001 to $.006(p<.05)$, VIF from 1.00 to 1.21 , Durbin-Watson $=1.00$. Therefore, it can be affirmed that the database is consistent with these three models. In model 1, $14.60 \%$ of the variation in SEIM exposure among adolescents is explained by sexual sensation seeking. In model 2, $17.80 \%$ of the variation in SEIM exposure among adolescents is explained by sexual sensation seeking and Internet usage time for entertainment. In model 3, 19.80\% of the variation in SEIM exposure among adolescents is explained by sexual sensation seeking, Internet usage time for entertainment, and perceived realism of SEIM. The regression coefficient for each model is shown in Table 3.

In particular, the variation of exposure to SEIM is most explained by sexual sensation seeking with $29.00 \%(p<.001)$, followed by Internet usage time for entertainment with $18.00 \%(p=.001)$, and perceived realism of SEIM with $16.00 \%(p=.006)$.

\section{DISCUSSION}

Hypothesis 1 was confirmed with the research findings that the majority of adolescents were exposed to SEIM, with more boys than girls being exposed to SEIM and the prevalence of passive exposure was higher than of active exposure. Our finding that male adolescents reported a higher level of SEIM exposure than female counterparts was consistent with previous research results (Flood, 2007; Lim et al., 2017; Peter \& Valkenburg, 2006a; Sabina, Wolak, \& Finkelhor, 2008). For types of exposure, our finding that the rate of adolescents who were passively exposed to SEIM was higher than those being involved in active exposure was in line with a study with Cambodian teenagers (Lopez et al., 2015). However, it is in contrast to a study of Taiwanese adolescents by Chen et al. (2013), which concluded that more teenagers were actively exposed than were passively exposed to SEIM (Chen et al., 2013).

Our study found personal psychological attributes including sexual sensation seeking, perceived realism of SEIM and Internet usage time for entertainment positively influenced adolescent exposure to SEIM. These results are in line with the previous literature indicating that greater exposure to SEIM was found among adolescents with a higher degree of sexual sensation seeking (Beyens et al., 2014; Luder et al., 2011; Ševčíková et al., 2014); and among those with more Internet usage time for entertainment (Mitchell et al., 2003; Rasmussen et al., 2015). Thus, hypothesis $\mathrm{H} 2$ is partially supported, confirming that sexual sensation seeking, Internet usage time for entertainment and perceived realism of SEIM are variables that positively predict SEIM exposure among adolescents. Meanwhile, hypothesis H3 is rejected; life satisfaction, family cohesion, and parental monitoring 
Table 3

Multivariate linear regression to predict scores of exposure to SEIM among adolescents

\begin{tabular}{|c|c|c|c|c|c|c|c|c|}
\hline \multicolumn{2}{|c|}{ Model } & \multicolumn{2}{|c|}{$\begin{array}{l}\text { Unstandardized } \\
\text { coefficients }\end{array}$} & \multirow{2}{*}{$\begin{array}{c}\text { Standardized } \\
\text { coefficients }\end{array}$} & \multirow[t]{2}{*}{$t$} & \multirow[t]{2}{*}{$p$} & \multicolumn{2}{|c|}{$\begin{array}{l}\text { Collinearity } \\
\text { statistics }\end{array}$} \\
\hline & & $B$ & $S E$ & & & & Tolerance & VIF \\
\hline \multirow[t]{2}{*}{1} & (Constant) & 1.65 & .22 & & 7.38 & $<.001$ & & \\
\hline & Sexual sensation seeking & 0.76 & .11 & .38 & 6.94 & $<.001$ & 1.00 & 1.00 \\
\hline \multirow[t]{3}{*}{2} & (Constant) & 1.31 & .24 & & 5.47 & $<.001$ & & \\
\hline & Sexual sensation seeking & 0.73 & .10 & .36 & 6.71 & $<.001$ & 0.99 & 1.01 \\
\hline & Internet use & 0.08 & .02 & .18 & 3.46 & .001 & 0.99 & 1.01 \\
\hline \multirow[t]{4}{*}{3} & (Constant) & 0.92 & .27 & & 3.36 & .001 & & \\
\hline & Sexual sensation seeking & 0.59 & .11 & .29 & 5.03 & $<.001$ & 0.82 & 1.21 \\
\hline & Internet use & 0.08 & .02 & .18 & 3.42 & .001 & 0.99 & 1.01 \\
\hline & Perceived realism of SEIM & 0.22 & .08 & .16 & 2.77 & .006 & 0.82 & 1.21 \\
\hline
\end{tabular}

Note. Dependent variable: exposure to SEIM; Durbin-Watson $=1.00$.

are not variables that negatively predict exposure to SEIM among teenagers.

The findings in this study are important for the design and organization of current sex and sexual education programs for adolescents. Currently, sexual education for children in Vietnam does not meet the needs of children to understand this issue because in the Confucian culture in Vietnam, sex and sexual education for children are considered as a taboo (Blanc, 2013). Schools neglect sexual education for children, and not only parents in the countryside but also parents in the city with high levels of education also find it difficult to educate sexuality for children (Ha, 2017). There is ambiguity in sexual education for children, and it is a fact that only a very small number of non-public elementary schools have sexual education programs for children while parents are ashamed and confused when educating sexuality at home (Sen, 2020). Sexual education in Vietnam tends to be on morality rather than on responsible decision-making (Efroymson, 1996). Currently, in the primary school curriculum, knowledge of sexual education is included in the 5th grade Science subject, in the subject of Humans and Health, but is still theoretical and academic (as cited in Nguyen, 2018).

The results of our research reveal that the majority of adolescents surveyed reported that they viewed and were exposed to SEIM. When sexual education in the family and school does not meet the needs of children, SEIM is considered an information channel to help children understand sexual health.

As an alternative to this information channel, a sexual education program and content for chil- dren should start at the beginning of primary school (Chi, 2018). The content of sexual education should focus on responsible decision making, not just on educating morality. The subject of sexual education for children should be diversified to include schools, parents and the community such as startups of young people, with the closeness and approach suitable for young people. For example, the startup We Grow Edu in their early 20s has an ambitious goal of empowering children and young people from the ages of five to 24 to better understand sex and sexuality (Nguyen, 2020). With the support of technology, the form of sexual education for children should mix between the orthodox way through lessons at school, at home and forms of online education, social networking sites, and communication channels (Ly, 2020).

The Government of Vietnam has issued Decree No.56/2017/ND-CP detailing a number of articles of the Law on Children, including Chapter IV devoted to "Responsibility to protect children in the internet environment", which clearly states that: "Agencies, organizations and individuals operating in the Internet environment shall coordinate with competent state agencies and other organizations and individuals in ensuring information security for children in the Internet environment; and prevent information that is harmful to children in accordance with the law on cyber information security" (GOV of Vietnam, 2017).

Our research results reveal that current laws do not comprehensively prevent adolescents from being exposed to SEIM. Therefore, technology-based measures such as Internet user age verification
Exposure to SEIM among adolescents 
software, Internet content monitoring software, together with parental monitoring of adolescents using the Internet, may contribute to reducing passive exposure to SEIM among adolescents, especially for young children.

\section{LIMITATION AND FUTURE STUDIES}

Luot V. Nguyen,

Thu H.Tran,

Thu T. A. Nguyen,

Dat B. Nguyen,

Harriot Beazley,

Mai T.T. Giang
The findings of our study were able to address the research hypotheses. However, this research still has certain limitations. This is a cross-sectional study and the exposure to SEIM is assessed at a time, not a process in a certain period of time. Another limitation was the use of self-reported data on exposure to SEIM by adolescents, which may have biased recall. The study used a convenient sampling method, and non-school samples were not selected. The school environment in which the study was conducted may have led students to be less truthful than if they had been asked to conduct the survey elsewhere.

Future research should focus on elucidating the relationship between SEIM exposure and risky sexual behaviors, particularly in the context of the increasing rates of unintended pregnancy and abortion among young Vietnamese (Hoang, Pham, Quach, Nguyen, \& Nguyen, 2012; MOHA \& UNFPA, 2015). Such research may help answer the question whether adolescents exposed to SEIM do not use condoms during sexual intercourse, resulting in unintended pregnancy. Additionally, future qualitative research with in-depth interviews and focus group discussions and other forms of qualitative research is suggested to deepen our understanding of the ways in which young people in Vietnam engage with SEIM, and their reasons for doing so.

\section{CONCLUSIONS}

In the context of sex and sexual education for children in Vietnam being heavily affected by the prohibition of Confucian culture, the majority of adolescents in this study have contact with SEIM both actively and passively. The evidence in this study suggests that parents, schools and other stakeholders should provide sexual education to children at earlier ages and diversify forms of sexual education for children. The content of sexual education should focus on responsible decision making among adolescents.

\section{ACKNOWLEDGEMENTS}

This research is funded by Vietnam National University, Hanoi (VNU) under project number QG.19.36.

\section{References}

Alexy, E. M., Burgess, A. W., \& Prentky, R. A. (2009). Pornography use as a risk marker for an aggressive pattern of behavior among sexually reactive children and adolescents. Journal of the American Psychiatric Nurses Association, 14, 442-453. https:// doi.org/10.1177/1078390308327137

Arrington-Sanders, R., Harper, G. W., Morgan, A., Ogunbajo, A., Trent, M., \& Fortenberry, J. D. (2015). The role of sexually explicit material in the sexual development of same-sex-attracted Black adolescent males. Archives of Sexual Behavior, 44, 597608. https://doi.org/10.1007/s10508-014-0416-x

Berk, L. E. (2017). Development through the lifespan (7th ed.). New York: Pearson.

Bélanger, D., \& Hong, K. T. (1999). Single women's experiences of sexual relationships and abortion in Hanoi, Vietnam. Reproductive Health Matters, 7, 7182. https://doi.org/10.1016/s0968-8080(99)90008-3

Beyens, I., Vandenbosch, L., \& Eggermont, S. (2014). Early adolescent boys' exposure to Internet pornography. The Journal of Early Adolescence, 35, 10451068. https://doi.org/10.1177/0272431614548069

Blanc, M. (2013). Sex education for Vietnamese adolescents in the context of the HIV/AIDS epidemic: The NGOs, the school, the family and the civil society. In E. Micollier (Ed.), Sexual cultures in East Asia. The social construction of sexuality and sexual risk in a time of AIDS (pp. 241-262). Abingdon: Taylor \& Francis.

Bleakley, A., Hennessy, M., \& Fishbein, M. (2011). A model of adolescents' seeking of sexual content in their media choices. Journal of Sex Research, 48, 309-315. https://doi.org/10.1080/00224499.2010.49 7985

Braun-Courville, D. K., \& Rojas, M. (2009). Exposure to sexually explicit Web sites and adolescent sexual attitudes and behaviors. Journal of Adolescent Health, 45, 156-162. https://doi.org/10.1016/j.jadohealth.2008.12.004

Bui, T. P. T., \& Nguyen, V. L. (2018). Sexual behaviors among young Vietnamese today. Paper presented at the Psychology and Sustainable Development Hanoi, Vietnam.

Chen, A. S., Leung, M., Chen, C. H., \& Yang, S. C. (2013). Exposure to Internet pornography among Taiwanese adolescents. Social Behavior and Personality, 4, 157-164. https://doi.org/10.2224/sbp.2013.41.1.157

Chi, M. (2018, December 27). Experts call for earlier sex education classes in schools. Retrieved from https://english.vietnamnet.vn

Cooper, A. L. (1998). Sexuality and the Internet: Surfing into the New Millennium. CyberPsychology \& Behavior, 1, 187-193. https://doi.org/10.1089/ cpb.1998.1.187

de Graaf, H., van de Schoot, R., Woertman, L., Hawk, S. T., \& Meeus, W. (2012). Family cohesion 
and romantic and sexual initiation: a three wave longitudinal study. Journal of Youth and Adolescence, 41, 583-592. https://doi.org/10.1007/s10964011-9708-9

Do, N. K., \& Barh, W. (2017). The changes in marital satisfaction across the first years of marriage among Vietnamese women. Proceedings of International Conference: Human Well-being and Sustainable Development, 1, 252-261.

Do, T. T., Khuat, H. T., \& Nguyen, A. T. V. (2017). More property, better sex? The relationship between property ownership and sexual satisfaction among married Vietnamese women. Sex Roles, 79, 409-420. https://doi.org/10.1007/s11199-0170879-z

Downing, M. J. Jr, Schrimshaw, E. W., Scheinmann, R., Antebi-Gruszka, N., \& Hirshfield, S. (2017). Sexually explicit media use by sexual identity: a comparative analysis of gay, bisexual, and heterosexual men in the United States. Archives of Sexual Behavior, 46, 1763-1776. https://doi.org/10.1007/ s10508-016-0837-9

Duong, C. T., Nguyen, T. H., Hoang, T. T. H., Nguyen, V. V., Do, T. M. N., Pham, V. H., \& Roger, D. (2004). Risky and bridging sexual behaviors among young people in Hai Phong. Hanoi: Medical Publishing House.

Efrati, Y., \& Amichai-Hamburger, Y. (2020). Are adolescents who consume pornography different from those who engaged in online sexual activities? Children and Youth Services Review, 111, 104843. https://doi.org/10.1016/j.childyouth.2020.104843

Efroymson, D. (1996). Vietnam faces modern sexuality problems with inadequate knowledge and solutions. SIECUS Report, 24, 4-6.

Flood, M. (2007). Exposure to pornography among youth in Australia. Journal of Sociology, 43, 45-60. https://doi.org/10.1177/1440783307073934

GOV of Vietnam (2017). Decree no. 56/2017/ND-CP detailing some articles of the Law on Children/the Law on Children. Hanoi: Government of the Socialist Republic of Vietnam.

Ha, T. (2017, March 28). Schools neglect teaching students about sex education. Retrieved from https:// english.vietnamnet.vn

Hardy, S. A., Steelman, M. A., Coyne, S. M., \& Ridge, R. D. (2013). Adolescent religiousness as a protective factor against pornography use. Journal of Applied Developmental Psychology, 34, 131-139. https://doi. org/10.1016/j.appdev.2012.12.002

Hoang, T. A., Pham, V. T., Quach, T. T., Nguyen, Q. P., \& Nguyen, T. T. (2012). What do they know? What do they want to know? What do we think they need to know? A research on adolescent sexuality and sexuality education. Hanoi: Center for Creative Initiative in Health and Population.

Holt, T. J., Bossler, A. M., \& May, D. C. (2012). Low self-control, deviant peer associations, and juve- nile cyber deviance. American Journal of Criminal Justice, 37, 378-395. https://doi.org/10.1007/s12103011-9117-3

Internet World Stats (2019). Top 20 countries with the highest number of internet users. Retrieved from https://www.internetworldstats.com/top20.htm

Kalichman, S. C., \& Rompa, D. (1995). Sexual Sensation Seeking and Sexual Compulsivity scales: Reliability, validity, and predicting HIV risk behavior. Journal of Personality Assessment, 65, 586-601. https://doi.org/10.1207/s15327752jpa6503_16

Khuat, H. T., Bach, D. L., \& Nguyen, H. N. (2009). Sexuality in contemporary Vietnam: Easy joke about but hard talk about. Hanoi: Knowledge Publisher and Vnn Publishing.

Kim, Y. H. (2001). Korean adolescents' health risk behaviors and their relationships with the selected psychological constructs. Journal of Adolescent Health, 29, 298-306. https://doi.org/10.1016/s1054139x(01)00218-x

Kim, Y. (2011). Adolescents' health behaviours and its associations with psychological variables. Central European Journal of Public Health, 19, 205-209. https://doi.org/10.21101/cejph.a3694

Lim, M. S. C., Agius, P. A., Carrotte, E. R., Vella, A. M., \& Hellard, M. E. (2017). Young Australians' use of pornography and associations with sexual risk behaviours. Australian and New Zealand Journal of Public Health, 41, 438-443. https://doi.org/ 10.1111/1753-6405.12678

Lo, V. H., \& Wei, R. (2005). Exposure to Internet pornography and Taiwanese adolescents' sexual attitudes and behavior. Journal of Broadcasting \& Electronic Media, 49, 221-237. https://doi.org/10.1207/ s15506878jobem4902_5

Lopez, J. R., Mukaire, P. E., \& Mataya, R. H. (2015). Characteristics of youth sexual and reproductive health and risky behaviors in two rural provinces of Cambodia. Reproductive Health, 12, article 83. https://doi.org/10.1186/s12978-015-0052-5

Luder, M. T., Pittet, I., Berchtold, A., Akre, C., Michaud, P. A., \& Suris, J. C. (2011). Associations between online pornography and sexual behavior among adolescents: Myth or reality? Archives of Sexual Behavior, 40, 1027-1035. https://doi. org/10.1007/s10508-010-9714-0

Ly, M. (2020, July 13). Nói thẳng "chuyện ấy", có gì phải bí mật [Frankly speaking "sex", there is nothing secret]. Retrieved from https://tuoitre.vn/

Ma, C. M., \& Shek, D. T. (2013). Consumption of pornographic materials in early adolescents in Hong Kong. Journal of Pediatric and Adolescent Gynecology, 26, S18-S25. https://doi.org/10.1016/j.jpag. 2013.03.011

Mai, G. T. T., Luot, N. V., Beazley, H., \& Dat, N. B. (2019). Self-esteem among "left-behind children" of labor migrant parents in rural Northern Vietnam. VNU Journal of Social Sciences and Humani-
Exposure to SEIM among adolescents 
ties, 5, 595-615. https://doi.org/10.33100/jossh5.5. GiangThiThanhMai.etal

Mesch, G. S. (2009). Social bonds and internet pornographic exposure among adolescents. Journal of Adolescence, 32, 601-618. https://doi.org/10.1016/j. adolescence.2008.06.004

Mitchell, K. J., Finkelhor, D., \& Wolak, J. (2003). The exposure of youth to unwanted sexual material on the Internet. Youth \& Society, 34, 330-358. https:// doi.org/10.1177/0044118x02250123

Luot V. Nguyen,

Thu H. Tran,

Thu T. A. Nguyen,

Dat B. Nguyen,

Harriot Beazley,

Mai T. T. Giang

MOHA \& UNFPA (2015). National report on Vietnamese youth. Hanoi: MOHA Vietnam \& UNFPA Vietnam.

Ngo, D. A., Ross, M. W., \& Ratliff, E. A. (2009). Influence of the Internet on sexual practice among adolescents in Hanoi, Vietnam. Hanoi: Thegioi Publishing.

Nguyen, H. T. (2018). Discourse on gender and sexual diversity in Vietnam: a crossroads of history, culture and institutions. VNU Journal of Social Sciences and Humanities, 4, 34-47.

Nguyen, H. T. (2019). From silicone and hormone injecting to sex reassignment surgery: The precarious road to becoming female of transgender funeral performers in Ho Chi Minh City, Vietnam. Culture, Health \& Sexuality, 21, 999-1011. https:// doi.org/10.1080/13691058.2018.1533144

Nguyen, M. G. (2018). Integrated teaching some contents of sex education for 3rd grade student. Ho Chi Minh University of Education Journal of Science, 15, 76-89.

Nguyen, S. (2020, May 8). The millennial changing the face of sex education in Vietnam. Retrieved from https://www.aljazeera.com

Nguyen, V. L., Bui, T. P. T., \& Le, N. H. A. (2017). Sexual communication and sexual satisfaction among youth's Vietnamese. Proceedings of International Conference: Human Well-being and Sustainable Development, 1, 12-50.

Owens, E.W., Behun, R. J., Manning, J. C., \& Reid, R. C. (2012). The impact of Internet pornography on adolescents: a review of the research. Sexual Addiction \& Compulsivity, 19, 99-122. https://doi.org/10. 1080/10720162.2012.660431

Peter, J., \& Valkenburg, P. M. (2006a). Adolescents' exposure to sexually explicit material on the Internet. Communication Research, 33, 178-204. https:// doi.org/10.1177/0093650205285369

Peter, J., \& Valkenburg, P. M. (2006b). Adolescents' exposure to sexually explicit online material and recreational attitudes toward sex. Journal of Communication, 56, 639-660. https://doi.org/10.1111/ j.1460-2466.2006.00313.x

Peter, J., \& Valkenburg, P. M. (2011a). The use of sexually explicit internet material and its antecedents: a longitudinal comparison of adolescents and adults. Archives of Sexual Behavior, 40, 1015-1025. https://doi.org/10.1007/s10508-010-9644-x

Peter, J., \& Valkenburg, P. M. (2011b). The influence of sexually explicit Internet material on sexual risk behavior: a comparison of adolescents and adults. Journal of Health Communication, 16, 750-765. https://doi.org/10.1080/10810730.2011.551996

Peter, J., \& Valkenburg, P. M. (2016). Adolescents and pornography: a review of 20 years of research. The Journal of Sex Research, 53, 509-531. https://doi. org/10.1080/00224499.2016.1143441

Pham, V. H. (2018). Culture of sexuality in Vietnam from $10^{\text {th }}$ to the $19^{\text {th }}$ century. Hanoi: Vietnam $\mathrm{Na}$ tional University Press.

Rasmussen, E. E., Ortiz, R. R., \& White, S. R. (2015). Emerging adults' responses to active mediation of pornography during adolescence. Journal of Children and Media, 9, 160-176. https://doi.org/10.1080/ 17482798.2014.997769

Rasmussen, K. R., \& Kohut, T. (2019). Does religious attendance moderate the connection between pornography consumption and attitudes toward women? Journal of Children and Media, 56, 38-49. https://doi.org/10.1080/00224499.2017.1396571

Sabina, C., Wolak, J., \& Finkelhor, D. (2008). The nature and dynamics of Internet pornography exposure for youth. CyberPsychology \& Behavior, 11, 691-693. https://doi.org/10.1089/cpb.2007.0179

Sen (2020). Dim light at the end of sex education tunnel. Retrieved from https://e.vnexpress.net

Ševčíková, A., Šerek, J., Barbovschi, M., \& Daneback, K. (2014). The roles of individual characteristics and liberalism in intentional and unintentional exposure to online sexual material among European youth: a multilevel approach. Sexuality Research and Social Policy, 11, 104-115. https://doi. org/10.1007/s13178-013-0141-6

Shek, D. T. L., \& Ma, C. M. S. (2012). Consumption of pornographic materials among early adolescents in Hong Kong: Profiles and psychosocial correlates. International Journal on Disability and Human Development, 11, 143-150. https://doi.org/ 10.1515/ijdhd-2012-0024

Shek, D. T. L., \& Ma, C. M. S. (2014). Using structural equation modeling to examine consumption of pornographic materials in Chinese adolescents in Hong Kong. International Journal on Disability and Human Development, 13, 239-245. https://doi. org/10.1515/ijdhd-2014-0309

Skoog, T., Stattin, H., \& Kerr, M. (2009). The role of pubertal timing in what adolescent boys do online. Journal of Research on Adolescence, 19, 1-7. https:// doi.org/10.1111/j.1532-7795.2009.00578.x

Socialist Republic of Vietnam (SRV) (2016). Decree no. 56/2017/ND-CP detailing some articles of the Law on Children/the Law on Children. The Law no. 102/2016/ QH13. Hanoi: Socialist Republic of Vietnam.

TNS (2014). Vietnam consumer behavior research 2014. Global Connected Consumer Study 2014.

Tomic, I., Buric, J., \& Stulhofer, A. (2018). Associations between Croatian adolescents' use of sexually explicit material and sexual behavior: Does parental 
monitoring play a role? Archives of Sexual Behavior, 47, 1881-1893. https://doi.org/10.1007/s10508017-1097-z

Tran, T. N., \& Nguyen, T. M. P. (2016). Attitudes, subjective norms, behavioral intentions, and sexting behaviors: Investigation in secondary school pupils and undergraduate students. VNU Journal of Social Sciences and Humanities, 2, 58-73.

Trinh, T. V. (2004). Communication between parents and adolescents about sexuality: Content, barriers and motives for communication. Hanoi: Medical Publishing House.

Truong, T. K. H. (2013). Development psychology. Hanoi: Vietnam National University Publishing.

Tsaliki, L. (2011). Playing with porn: Greek children's explorations in pornography. Sex Education, 11, 293-302. https://doi.org/10.1080/14681811.2011.59 0087

Van Ouytsel, J., Ponnet, K., \& Walrave, M. (2014). The associations between adolescents' consumption of pornography and music videos and their sexting behavior. Cyberpsychology, Behavior, and Social Networking, 17, 772-778. https://doi.org/10.1089/ cyber.2014.0365

Vandenbosch, L., \& Eggermont, S. (2013). Sexualization of adolescent boys. Men and Masculinities, 16, 283-306. https://doi.org/10.1177/1097184x13477866

Weber, M., Quiring, O., \& Daschmann, G. (2012). Peers, parents and pornography: Exploring adolescents' exposure to sexually explicit material and its developmental correlates. Sexuality \& Culture, 16, 408-427. https://doi.org/10.1007/s12119-012-9132-7

WHO (2018). Adolescent health in the South-East Asia Region. Retrieved from https://www.who.int/southeastasia/health-topics/adolescent-health?fbclid=I wAR04IS9ZqrVhKVcVgVyOSjR0ChzxQ9PJ_hFkvePye6ymzd5umqs1AmNogY0

Wight, D., Williamson, L., \& Henderson, M. (2006). Parental influences on young people's sexual behaviour: a longitudinal analysis. Journal of Adolescence, 29, 473-494. https://doi.org/10.1016/j.adolescence.2005.08.007

Wolak, J., Mitchell, K., \& Finkelhor, D. (2007). Unwanted and wanted exposure to online pornography in a national sample of youth Internet users. Pediatrics, 119, 247-257. https://doi.org/10.1542/ peds.2006-1891

Ybarra, M. L., \& Mitchell, K. J. (2005). Exposure to Internet pornography among children and adolescents: a national survey. CyberPsychology \& Behavior, 8, 473-486. https://doi.org/10.1089/cpb.2005.8.473 